\title{
Detection of Gestational Diabetes Mellitus and Influence on Perinatal Outcomes from B-Mode Ultrasound Images Using Deep Neural Network
}

\author{
Yuhui Liu $\mathbb{D}^{1},{ }^{1}$ Yu Wang $\mathbb{D}{ }^{2}$ Yang Zhang $\mathbb{D}^{3},{ }^{3}$ and Rulei Cheng $\mathbb{C}^{3}$ \\ ${ }^{1}$ Department of Ultrasound, Yangpu Economic Development Zone Hospital, Yangpu Economic Development Zone, Danzhou, \\ Hainan 578101, China \\ ${ }^{2}$ Department of Ultrasound, Zaozhuang Mining Group Central Hospital, Xuecheng District, Zaozhuang 277800, \\ Shandong, China \\ ${ }^{3}$ Department of Pathology, Suzhou Municipal Hospital Headquarters, Suzhou 215000, China
}

Correspondence should be addressed to Yuhui Liu; 189040046@stu.just.edu.cn

Received 6 October 2021; Revised 5 November 2021; Accepted 7 November 2021; Published 30 November 2021

Academic Editor: M Pallikonda Rajasekaran

Copyright (c) 2021 Yuhui Liu et al. This is an open access article distributed under the Creative Commons Attribution License, which permits unrestricted use, distribution, and reproduction in any medium, provided the original work is properly cited.

\begin{abstract}
The study was intended to explore the risk factors of gestational diabetes mellitus (GDM) and their influence on perinatal outcomes through deep neural network (DNN)-based Doppler color ultrasound (B-mode ultrasound) images. Specifically, 75 women with GDM were selected as the experimental group, and 75 healthy pregnant women were selected as the control (Ctrl) group. DNN uses the unsupervised method to pretrain layer by layer and then uses the supervised method to accumulate parameters of each layer, which can obtain good performance. In this study, the risk factors of GDM and their influence on the perinatal outcomes were analyzed by DNN-based B-mode ultrasound images. It was found that pregnancy age was a risk factor for $\operatorname{GDM}(\mathrm{OR}=2.566)$, preference for sweets was a risk factor for GDM $(\mathrm{OR}=1.678, P<0.001)$, and family history of DM was also a risk factor for $\mathrm{GDM}(\mathrm{OR}=12.789, P<0.001)$. The incidence of complications in the experimental group was higher than that in the Ctrl group $(P<0.05)$. Therefore, the true positive recognition (TPR) rate of DNN was significantly higher than that of the traditional method, and the pregnancy age, the preference for sweets before pregnancy, and the family history of DM may be risk factors for GDM; also, GDM was an influencing factor for pregnancy outcome, neonatal outcome, and complications.
\end{abstract}

\section{Introduction}

Diabetes mellitus (DM) is a type of metabolic type disease caused by a variety of pathogenic factors, characterized by hyperglycemia. Long-term hyperglycemia will damage the function of multiple systems [1]. GDM is a type of DM that only appears during pregnancy. Before pregnancy, the patient's glucose metabolism is normal [2]. The incidence of GDM around the world is about $1 \%$ to $14 \%$, and that in China is about $1 \%$ to $5 \%$, accounting for about $80 \%$ of pregnant women with DM, and it has increased in recent years [3]. GDM will weaken fetal heart function and cause neonatal hypoxia or even asphyxiation [4]. The clinical treatment process of pregnant women with DM is very complicated, which is risky for both mothers and children. Therefore,
GDM risk factors should be explored to reduce the occurrence of complications suffered by perinatal pregnant women and newborns [5]. In the study, Doppler ultrasound was adopted to detect GDM, analyze its risk factors, and explore the influence of these risk factors on the perinatal outcomes.

Deep learning is a type of machine learning algorithm that can gradually extract high-level features from the original input layer by layer. In image processing, the lower layer can identify the edges, while the higher layer can identify the parts that are meaningful to humans [6]. DNN was considered unable to train efficiently for a long time in the past [7]. In recent years, under the continuous research of Liu and Liu [8], DNN has gradually become popular. The unsupervised method is used to pretrain layer by layer, and then the parameters of each layer are piled up in a supervised 
way, which can achieve good performance when applied to B-mode ultrasound.

Some scholars believe that the age of pregnant women is related to the occurrence of GDM [9]. Dantas et al. [10] found that after pregnancy, the pregnancy age, preference for sweets, and family history of DM were risk factors for GDM. Kim et al. [11] found that as women's age at first pregnancy increased, the probability of GDM increased, and age at first pregnancy was one of the independent risk factors for GDM. Relevant clinical data reveal that GDM will seriously affect perinatal outcomes. The incidence of polyhydramnios, pregnancy hypertension, and cesarean section in GDM women is higher than that of normal pregnant women [12], and GDM may cause fetal malformations, macrosomia, and even death [13].

There are many studies on GDM risk factors, but the influence of GDM risk factors on the perinatal outcome remains to be explored. In this study, the pregnancy characteristics data of pregnant women were collected. The innovation of this study was to understand the clinical pregnancy characteristics of hospitalized pregnant women by constructing DNN-based B-mode ultrasound images and to analyze the risk factors of GDM and its influence on perinatal outcome. It was hoped that the results of this study can provide a good reference for early intervention on the risk factors of GDM and reduce the impact of GDM on pregnant women and newborns.

\section{Materials and Methods}

2.1. Selection of Research Samples. In this study, 75 women with GDM who visited the hospital from November 18, 2018, to October 20,2019, were selected as the experimental group, and 75 healthy pregnant women were selected as the Ctrl group. The study has got permission from the medical ethics committee of the hospital, and the patients and their families have signed informed consent forms.

Inclusion criteria were as follows: (i) patients diagnosed with GDM; (ii) primiparas with singleton pregnancy; (iii) patients not taking drugs, which interfere with metabolism and lipid metabolism; (iv) patients younger than 45 years of age; and (v) pregnant women with clear consciousness and normal cooperation in research.

Exclusion criteria were as follows: (i) patients with mental illness; (ii) patients with hypertension and kidney, cardiovascular, and other diseases; (iii) patients who smoked or drank alcohol; and (iv) patients who withdrew from the experiment halfway.

2.2. Observation Indicators. The information of patient's height, age, weight, residence, education level, occupation, and DM family history was recorded. The observation indexes of pregnancy include number of pregnancies, number of abortions, premature births, and complications of pregnancy. The number of pregnancies refers to the number of intrauterine pregnancies, including abortion, premature delivery, and full-term delivery. Abortion refers to the pregnancy less than 28 weeks or termination of pregnancy because of fetal weight less than $1 \mathrm{~kg}$. Premature birth is defined as delivery between 28 and 37 weeks of gestation. A full-term delivery refers to a delivery between 37 and 42 weeks of gestation. Pregnancy complications are those associated with GDM, such as pregnancy-induced hypertension and pregnancy-associated heart disease. The indexes for perinatal outcomes include delivery method and postpartum hemorrhage, and delivery methods include cesarean section, vaginal delivery, and assisted vaginal delivery. The clinical characteristics of newborns included gestational age, height, weight, and neonatal complications. Gestational age refers to the gestational age at birth; newborns with a weight greater than $4000 \mathrm{~g}$ are considered to be giant babies.

2.3. B-Mode Ultrasound Examination Method. In this study, an ultrasonic machine was used, and the probe frequency was $3.5 \sim 5.0 \mathrm{MHz}$. The pregnant woman took the supine position. Then, the doctor asked information about ultrasound diagnosis results during early pregnancy and last menstruation to determine the pregnancy cycle. Then, the conventional growth indicators including head circumference, double parietal diameter, and femur and abdominal circumference of the fetus were measured. When it was confirmed that there were no special problems, B-mode ultrasound was performed to measure venous blood flow. After the fetal pulmonary venous catheter blood flow images, fetal venous catheter blood flow images, and fetal inferior vena cava blood flow images were obtained, the peak ventricular systolic velocity (S), ventricular diastolic peak velocity (D), and atrial systolic maximum velocity (A) were measured, and the ratio of systolic to diastolic velocity (S/D) and pulsatility index (PI) were calculated. $\mathrm{PI}=$ (systolic peak velocity - end-diastolic blood flow velocity)/average blood flow velocity. The time from the stopping point of the blood flow spectrum to the next starting point of the blood flow spectrum in diastolic phase of the mitral valve orifice $\left(t_{1}\right)$ and the duration of the blood flow spectrum in the systolic phase of the aortic valve orifice $\left(t_{2}\right)$ were measured. Tei index $=\left(t_{1}-t_{2}\right) / t_{2}$. The fetal renal artery resistance index $(\mathrm{RI})$ was calculated as follows. $\mathrm{RI}=$ (peak systolic velocity - enddiastolic blood flow velocity)/peak systolic velocity. At the same time, the early diastolic notch was observed.

2.4. DNN Working Mechanism. DNN is an important backpropagation algorithm, which is essentially the same as BP neural algorithm. The main difference lies in full connection or incomplete connection. Full connection only needs to link the neurons of the first layer with the neurons of the adjacent two layers, but incomplete connection requires specific parameters before it connects each neuron.

Assuming that the convolutional layer is layer I, layer I-1 is the input layer, and layer I is calculated as follows:

$$
a_{j}^{I}=g\left[\sum_{i \in N_{j}} a_{i}^{I-1} \cdot b_{i j}^{I}+c_{j}^{I}\right],
$$

where $a_{j}^{I}$ is the $j$ feature mapping in layer $\mathrm{I}, N_{j}$ is the set of input feature mapping, and $b_{i j}^{I}$ is the $j$ th kernel in layer $I$. 
When the bias parameter is added, the activation function is input. Assuming that pooling layer is layer $I$, layer $I-1$ is set as the convolution layer, and then, the layer $I$ is calculated as follows.

$$
a_{j}^{I}=g\left(\omega_{j}^{I} \operatorname{deep}\left(a_{i}^{I-1}\right)+c_{j}^{I}\right),
$$

in which deep $\left(a_{i}^{I-1}\right)$ represents the downsampling function and $\omega$ represents the weight; generally, it is $1 / n^{2}$. According to backpropagation algorithm, when layer $\mathrm{I}$ is set as the convolution layer and layer $\mathrm{I}+1$ is the pooling layer, the whole area of the layer I is associated with the neurons in layer $I+1$, and the sensitivity of each neuron is calculated as follows:

$$
\delta_{j}^{I}=\omega_{j}^{I+1}\left(\dot{g}\left(a_{j}^{I}\right) \circ u p\left(\delta_{j}^{I+1}\right)\right),
$$

in which $\delta_{j}^{I}$ is the sensitivity of the neuron and $\omega$ is the weight. The sensitivities of all neurons in layer I feature map are summed up. The final result is the bias derivative in each feature map. The specific equation is as follows:

$$
\frac{\partial E}{\partial b_{j}}=\sum_{y, z}\left(\delta_{j}^{I}\right)_{y, z},
$$

in which $\partial E / \partial b_{j}$ is the bias derivative and $\delta_{j}^{I}$ is the sensitivity of the neuron. The basic idea of gradient descent algorithm is to update the model parameters based on the opposite direction of the gradient of the objective function, so as to obtain the minimum objective function. If $f(\beta)$ is the function to fit, then $h(\beta)$ is the loss function, and $\beta$ is the parameter to solve. The specific equation is as follows:

$$
\begin{aligned}
& f(\beta)=\sum_{j=0}^{m} \beta_{j} a_{j}, \\
& h(\beta)=\frac{1}{2 n} \sum_{i=1}^{n}\left(y_{i}-f_{\beta}\left(x^{i}\right)\right)^{2},
\end{aligned}
$$

in which $n$ is the sample in training set and $j$ refers to the number of parameters. The batch gradient descent method is used to solve it. The partial derivative of the loss function to the parameter is calculated, so as to obtain the gradient corresponding to each function. The equation is as follows:

$$
\frac{\partial h(\beta)}{\partial \beta_{j}}=-\frac{1}{n} \sum_{i=1}^{n}\left(y^{i}-f_{\beta}\left(x^{i}\right)\right) x_{j}^{i},
$$

in which $\beta$ is the parameter, $h(\beta)$ is the loss function, and $f(\beta)$ is the fitting function. $\beta$ is calculated as follows:

$$
\beta_{j}=\beta_{j}+\frac{1}{n} \sum_{i=1}^{n}\left(y^{i}-f_{\beta}\left(x^{i}\right)\right) x_{j}^{i} .
$$

To obtain the global optimal solution, all data of samples should be included in each calculation. If the amount of data is too large, it will affect the whole speed calculation and reduce the efficiency. Therefore, the stochastic gradient descent method is introduced for improvement. If the random gradient descent method is used to solve the parameters, the loss function calculates a single sample, but not all samples. The equation is as follows:

$$
h(\beta)=\frac{1}{n} \sum_{i=1}^{n}\left(y^{i}-f_{\beta}\left(x^{i}\right)\right)^{2} .
$$

Then, the partial derivative of the target variable in loss function $h(\beta)$ is calculated to update the corresponding gradient as follows:

$$
\beta_{j}=\beta_{j}+\left(y^{i}-f_{\beta}\left(x^{i}\right)\right) x_{j}^{i},
$$

in which $\beta$ is the parameter and $f(\beta)$ is the fitting function. The random gradient descent method is adopted to update the input sample data in real time. More corresponding sample data indicate more times of updating.

2.5. Sample Training Conditions and Test Indicators. Hardware environment for training was as follows: operating system-Ubuntu 14.0464 bit; CPU-Intel ${ }^{\circledR}$ Xeon (R) CPU E5-1630 v3 @ 3.70 GHz $\times 4$; memory: 64G; and GPU-Quadro K2200. Deep learning-based python version network framework is used, which can be accelerated by GPU. VOC2007 database is adopted for pretraining. At first, opencv dynamic library is employed to draw the bounding box of the target rectangle, then the $\mathrm{xml}$ file is fabricated and saved in Annotations, the training sample pictures are put in JPEG Images, and the folder corresponding to VOC2007 database is covered, and finally the sample size is trained.

Test Indicators. True positive (TP) indicates the number of correctly identified papillary thyroid carcinoma. TPR indicates the recognition rate of true positive. False negative (FN) indicates the number of thyroid papillary carcinoma that has not been correctly identified. FNR represents the recognition rate of false negatives.

2.6. Statistics. The data were processed by SPSS 20.0. The measurement data conforming to the normal distribution were calculated as mean \pm standard deviation $(\bar{x} \pm s)$, and the non-conforming count data were expressed by the percentage (\%). The independent sample $t$-test was adopted for measurement data of single factor analysis, and $\chi^{2}$ test was adopted for the comparison between groups. $P<0.05$ indicated that the difference was statistically significant, and and the logistic regression method was used for multivariate analysis of the data.

\section{Results}

3.1. Performance Comparison between Traditional Method and DNN. Table 1 shows that the false negative recognition rate of DNN was similar to that of artificial phase, reaching $25.6 \%$ and $23.1 \%$, respectively. The reason was that the structure of GDM image was complex and some texture features are difficult to distinguish. As for the true positive recognition rate, the probability of DNN was significantly higher than that of the traditional method, and the difference was statistically significant $(P<0.05)$. Therefore, it was of 
TABLE 1: Performance comparison between the traditional method and DNN.

\begin{tabular}{lcccc}
\hline & TP & TPR & FN & FNR \\
\hline Traditional method & 165 & 0.725 & 58 & 0.256 \\
DNN & 174 & $0.762^{*}$ & 54 & 0.231 \\
\hline
\end{tabular}

Note. ${ }^{*}$ indicates that compared with the traditional method, the difference is statistically significant $(P<0.05)$.

practical significance to apply DNN algorithm to the recognition of GDM ultrasound images.

3.2. The Basic Information. Univariate analysis of patients' occupation type, age distribution, place of residence, preference for sweets, bad habits, and DM family history found that there was no notable difference in occupation type and residence $(P>0.05)$ (Table 2$)$, while the difference between the two was notable in terms of the age distribution, preference for sweets, bad habits, and family history of DM $(P<0.05)$.

3.3. The Fetal Ultrasound Image of GDM Patients. B-mode ultrasound is a new method to detect the hemodynamics of fetuses of GDM patients. Fetal pulmonary venous catheter blood flow (Figure 1), venous catheter blood flow (Figure 2), and inferior vena cava blood flow are all composed of " 2 peaks and 1 valley". The first peak was the ventricular contraction peak (Peak S), which is the atrial pressure caused by caused by the ventricular diastole and the downward movement of the atrioventricular annulus during the contraction of the ventricle. The second peak is the ventricular diastolic peak (Peak D). After ventricular diastole, the atrial blood flows rapidly into the ventricle, reducing the filling pressure in the atrium, and the venous blood flows into the atrium rapidly for the second time. Valley 1 is the atrial contraction valley (Valley A), which is caused by the contraction of the atrium. Valley A of the pulmonary venous catheter and the venous catheter is a positive phase, and that of the inferior vena cava is a negative phase.

3.4. The Multivariate Logistic Regression Statistics Analysis of $G D M$. In univariate analysis, there was a notable difference in terms of preference for sweets, pregnancy age, and DM family history $(P<0.05)$. The logistic regression analysis revealed that pregnancy age was a risk factor for GDM $(\mathrm{OR}=2.566)$; preference for sweets was a risk factor for GDM ( $\mathrm{OR}=1.678)$, and family history of $\mathrm{DM}$ was a risk factor for GDM $(\mathrm{OR}=12.789)$ (Figure 3).

3.5. The Pulmonary Venous Blood Flow Parameters. There was no notable difference in $S$ and $D$ values between the two groups $(P>0.05)$ (Figure 4$)$. In contrast with Ctrl, the fetal pulmonary venous PI and Tei index were higher in the experimental group $(P<0.05)$ (Figure 5).
3.6. The Venous Catheter Blood Flow and Inferior Vena Cava Blood Flow Parameters of Fetuses. There was no notable difference in venous catheter blood flow and ventricular systolic peak velocity $(S)$, ventricular diastolic peak velocity (D), and atrial systole maximum flow rate $(A)$ between the two groups $(P>0.05)$ (Figures 6 and 7$)$.

3.7. The Fetal Renal Artery Hemodynamic Index. The renal artery RI and $S / D$ in the experimental group were higher than those in the Ctrl $(P<0.05)$ (Figure 8).

3.8. Comparison of Pregnancy Outcomes and Complications. The incidence of pregnancy hypertension, polyhydramnios, cesarean section, and complications in the experimental group was higher than that in the Ctrl group $(P<0.05)$ (Figure 9). Among them, the incidence of polyhydramnios of GDM women was $70.1 \%$, while that of normal women was $23 \%$.

3.9. Comparison of Neonatal Outcomes and Complications. Comparing the average gestational weeks of newborns in the experimental group with the average gestational weeks of newborns in the control group, it was found that the average gestational weeks of newborns in the experimental group were shorter, but the difference was not statistically significant $(P>0.05)$ (Figure 10); the average weight was higher $(P<0.05)$ (Figure 11); the incidence of macrosomia in the experimental group was higher $(12 \%$ vs. $6 \%)(P<0.05)$. There was no notable difference in polycythemia $(P>0.05)$. However, in terms of neonatal asphyxia, intrauterine distress, polycythemia, hypoglycemia, stillbirth, and jaundice, there was a notable difference $(P<0.05)$.

\section{Discussion}

GDM is a type of DM that only occurs during a specific period. It is a metabolic disease that may be inherited. Hod et al. [14] studied the risk factors for the onset of GDM and believed that it may be related to the weight of the pregnant woman, age at pregnancy, family history of DM, bad habits, and education degree.

DNN is the most suitable method for image feature extraction. At present, DNN has achieved remarkable results in target detection, and it has been applied to GDM ultrasound images [15]. The TPR of DNN was significantly higher than that of the traditional method, and the difference was statistically significant $(P<0.05)$. Therefore, it is of practical significance to apply DNN algorithm to the recognition of GDM ultrasound images. Logistic regression analysis showed that the regression coefficient was 0.967 $(\mathrm{OR}=2.566)$, which indicated that the older the age, the higher the incidence of GDM. This was consistent with the result that Onmez et al. [16] who pointed out that age was one of the independent risk factors for GDM. If pregnant women like to eat sweets before pregnancy, this may be a factor in the occurrence of GDM. In the study, it was found that regular intake of high-sugar foods in pregnant women 
TABLe 2: The basic information.

\begin{tabular}{|c|c|c|c|c|c|}
\hline Basic information & Characteristics & $\operatorname{Ctrl}(n=75)$ & Experimental group $(n=75)$ & $\chi^{2}$ & $P$ \\
\hline Age distribution & $\begin{array}{c}\text { Under } 35 \\
\text { Over } 35 \text { years old }\end{array}$ & $\begin{array}{l}22 \\
53\end{array}$ & $\begin{array}{l}18 \\
57\end{array}$ & 37.8 & 0.000 \\
\hline Type of occupation & $\begin{array}{c}\text { Physical strength } \\
\text { Brainpower }\end{array}$ & $\begin{array}{c}9 \\
66 \\
\end{array}$ & $\begin{array}{l}12 \\
63 \\
\end{array}$ & 3.786 & 0.846 \\
\hline Place of residence & $\begin{array}{l}\text { Urban area } \\
\text { Rural area } \\
\end{array}$ & $\begin{array}{l}21 \\
54 \\
\end{array}$ & $\begin{array}{l}15 \\
60 \\
\end{array}$ & 1.396 & 0.278 \\
\hline Sweets & $\begin{array}{c}\text { Like } \\
\text { Dislike }\end{array}$ & $\begin{array}{l}19 \\
56 \\
\end{array}$ & $\begin{array}{l}48 \\
27 \\
\end{array}$ & 36.683 & 0.015 \\
\hline Habits & $\begin{array}{c}\text { Good } \\
\text { Bad }\end{array}$ & $\begin{array}{l}35 \\
40\end{array}$ & $\begin{array}{l}10 \\
65\end{array}$ & 7.576 & 0.007 \\
\hline DM family history & $\begin{array}{l}\text { Yes } \\
\text { No }\end{array}$ & $\begin{array}{c}8 \\
67 \\
\end{array}$ & $\begin{array}{l}37 \\
38 \\
\end{array}$ & 6.198 & 0.035 \\
\hline
\end{tabular}

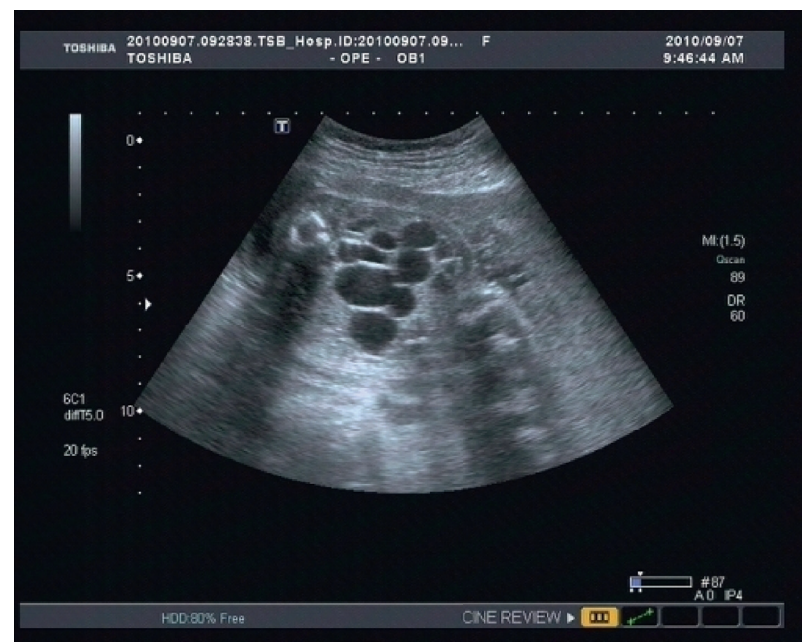

Figure 1: The pulmonary vein blood flow image of a fetus.

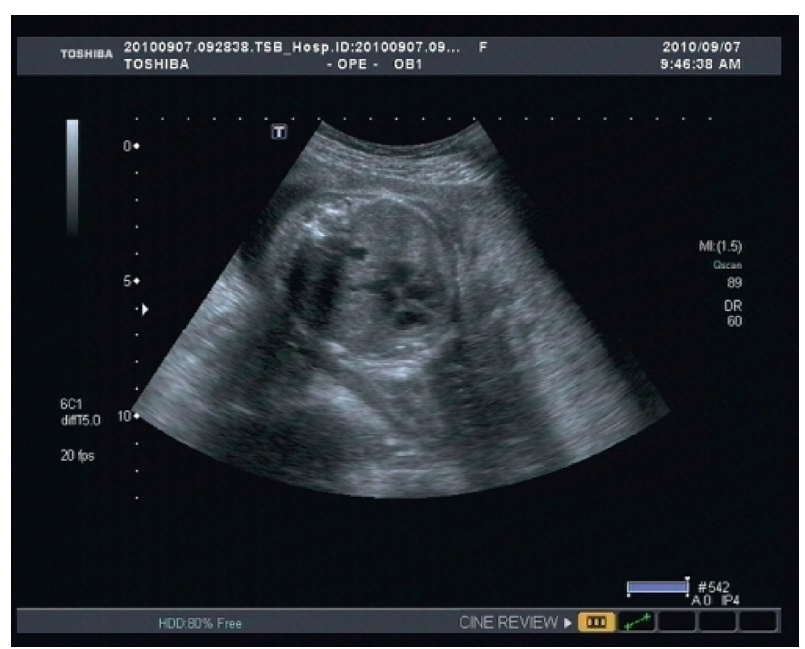

Figure 2: The venous catheter blood flow image of a fetus.

increased the probability of GDM $(\mathrm{OR}=1.678)$. Lee et al. [17] pointed out that whether the patient had a family history of DM was a risk factor for the occurrence of GDM,

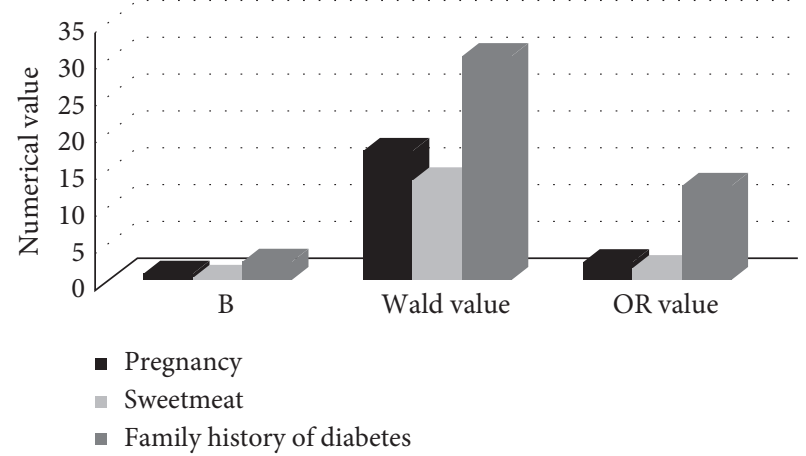

FIgURE 3: The multivariate logistic regression analysis of GDM. Note: B represents regression coefficient and intercept; OR indicates the odds ratio.

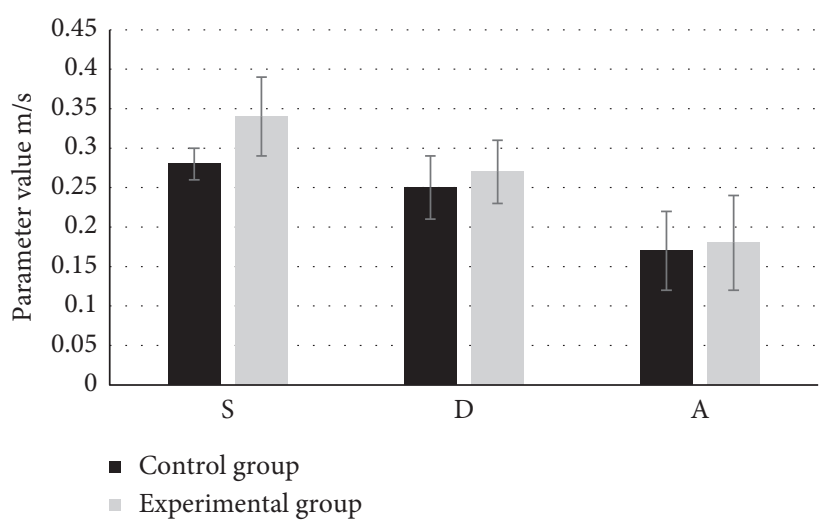

FIgURE 4: The fetal pulmonary venous blood flow parameters.

which was consistent with the results in the study that the family history of DM was positively correlated with the occurrence of GDM $(\mathrm{OR}=12.789)$. The regression coefficient was 2.567. Therefore, the family history of DM was one of the important risk factors for GDM. According to relevant research reports, the incidence of polyhydramnios in GDM pregnant women is 10 times higher than that of normal pregnant women $[18,19]$. In this study, the incidence of polyhydramnios in GDM pregnant women was $70.1 \%$, while 




Figure 5: The comparison of PI and Tei index. Note: in contrast with the Ctrl, ${ }^{*} p<0.05$ showed notable differences (with the same meaning below).

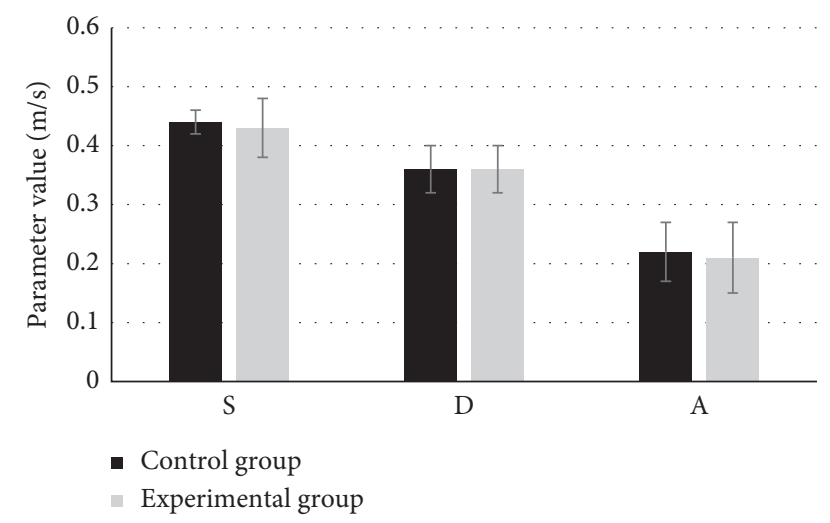

Figure 6: Comparison of fetal venous catheter blood flow parameters.

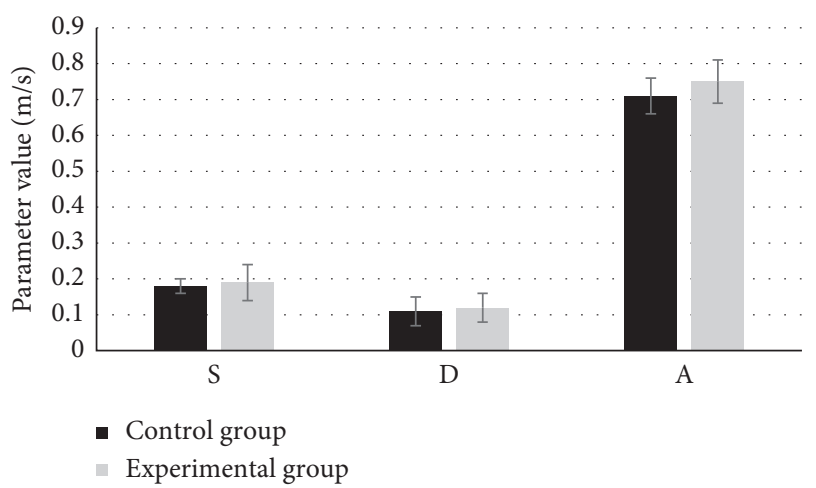

Figure 7: Comparison of fetal inferior vena cava blood flow parameters.

that of normal pregnant women was $23 \%$. It may be that GDM causes hyperglycemia in fetuses. As a result, hypertonic diuretic hormone secretion is strong, and fetal urination increases, which leads to polyhydramnios [20-22]. Under the stimulation of hyperglycemia, the protein synthesis amount increases, and the birth rate of giant babies increases. There is a study claiming that the incidence of macrophages as high as $60 \%$ due to GDM [23-25]. In this



FIgure 8: Comparison of fetal renal artery hemodynamic index.

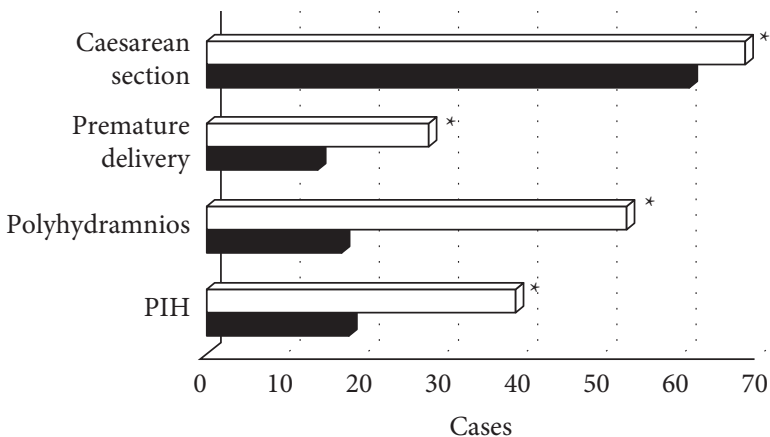

$\square$ Experimental group

- Control group

FIGURE 9: Comparison of pregnancy outcomes and complications.

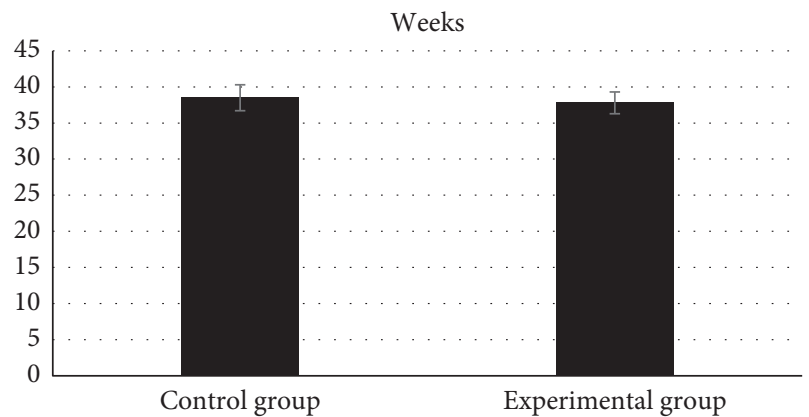

FIgURE 10: Comparison of the average gestational weeks.

study, the incidence of macrosomia in the Ctrl and the experimental groups was $6 \%$ and $12 \%$, respectively $(P<0.05)$.

To exclude the mixed effects of various factors, a multifactor statistical analysis was conducted to find out the factor with biggest influence. In the univariate analysis of GDM risk factors, the age of pregnant women, sweet food, and family history of DM were introduced into the regression equation, which was in line with the expected results of this study. 


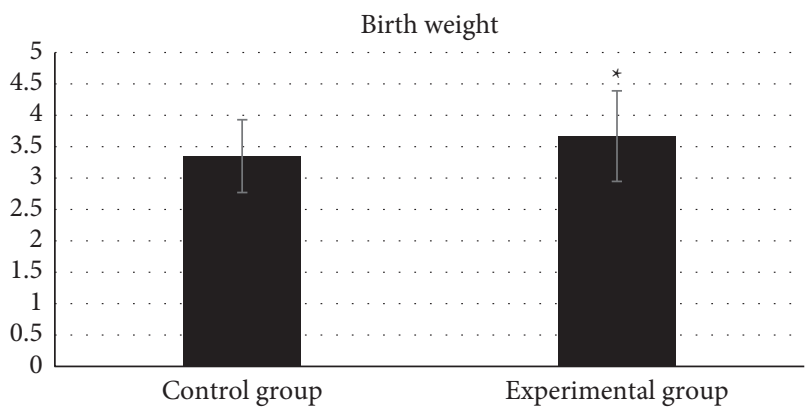

Figure 11: Comparison of birth weight of newborns.

\section{Conclusion}

After analysis of GDM risk factors and perinatal outcomes through DNN-based B-mode ultrasound images, it was found that the TPR rate of DNN was significantly higher than that of the traditional method, and pregnant women's age at pregnancy, prepregnancy preference for sweets, and family history of DM may be independent risk factors for GDM. GDM is an influence factor of pregnancy hypertension, polyhydramnios, and cesarean section. It is also an influence factor in neonatal birth weight, gestational weeks, macrosomia, neonatal asphyxia, intrauterine distress, polycythemia, and hypoglycemia. However, the number of samples is small, which may cause deviations in results. In the follow-up studies, the number of samples will be increased for further research. In conclusion, the results of the study provide a theoretical basis for early intervention of GDM risk factors, so as to reduce the impact of GDM on pregnant women and newborns.

\section{Data Availability}

The data used to support the findings of this study are available from the corresponding author upon request.

\section{Conflicts of Interest}

The authors declare that they have no conflicts of interest.

\section{References}

[1] M. Noory, J. F. Renz, P. L. Rosen, H. Patel, A. Schwartzman, and R. W. G. Gruessner, "Real-time, intraoperative Doppler/ ultrasound monitoring of islet infusion during total pancreatectomy with islet autotransplant: a first report," Transplantation Proceedings, vol. 51, no. 10, pp. 3428-3430, 2019.

[2] Y. Yu, R. Xie, C. Shen, and L. Shu, "Effect of exercise during pregnancy to prevent gestational diabetes mellitus: a systematic review and meta-analysis," Journal of Maternal-Fetal and Neonatal Medicine, vol. 31, no. 12, pp. 1632-1637, 2018.

[3] B. Daly, K. A. Toulis, N. Thomas et al., "Increased risk of ischemic heart disease, hypertension, and type 2 diabetes in women with previous gestational diabetes mellitus, a target group in general practice for preventive interventions: a population-based cohort study," PLoS Medicine, vol. 15, no. 1, Article ID e1002488, 2018.

[4] A. Ruohomäki, E. Toffol, S. Upadhyaya et al., "The association between gestational diabetes mellitus and postpartum depressive symptomatology: a prospective cohort study," Journal of Affective Disorders, vol. 241, pp. 263-268, 2018.

[5] K. A. Okesene-Gafa, A. E. Moore, V. Jordan, L. Mc Cowan, and C. Crowther, "Probiotic treatment for women with gestational diabetes to improve maternal and infant health and well-being," Cochrane Database of Systematic Reviews, vol. 6, no. 6, Article ID CD012970, 2020.

[6] M. Hu, Y. Zhong, S. Xie, H. Lv, and Z. Lv, "Fuzzy system based medical image processing for brain disease prediction," Frontiers in Neuroscience, vol. 15, Article ID 714318, 2021.

[7] Z. Wan, Y. Dong, Z. Yu, H. Lv, and Z. Lv, "Semi-supervised support vector machine for digital twins based brain image fusion," Frontiers in Neuroscience, vol. 15, Article ID 705323, 2021.

[8] H. Liu and J. Liu, "Improved support vector machine algorithm based on the influence of Gestational Diabetes Mellitus on the outcome of perinatal outcome by ultrasound imaging," Pakistan journal of medical sciences, vol. 37, no. 6, pp. 1625-1629, 2021.

[9] A. B. Peixoto, N. J. M. Bravo-Valenzuela, W. P. Martins et al., "Impact of type I and type II maternal diabetes mellitus on fetal cardiac function assessment parameters using spectral and tissue Doppler," The International Journal of Cardiovascular Imaging, vol. 36, no. 7, pp. 1237-1247, 2020.

[10] A. M. A. Dantas, A. B. S. Palmieri, M. R. Vieira, M. L. R Souza, and J. C Silva, "Doppler ultrasonographic assessment of fetal middle cerebral artery peak systolic velocity in gestational diabetes mellitus," International Journal of Gynaecology and Obstetrics: The Official Organ of the International Federation of Gynaecology and Obstetrics, vol. 144, no. 2, pp. 174-179, 2019.

[11] Y.-H. Kim, J.-H. Kim, and C. Park, "Evaluation of tissue Doppler ultrasonographic and strain imaging for assessment of myocardial dysfunction in dogs with type 1 diabetes mellitus," American Journal of Veterinary Research, vol. 79, no. 10, pp. 1035-1043, 2018.

[12] A. Kijmanawat, P. Panburana, S. Reutrakul, and C. Tangshewinsirikul, "Effects of probiotic supplements on insulin resistance in gestational diabetes mellitus: a doubleblind randomized controlled trial," Journal of Diabetes Investigation, vol. 10, no. 1, pp. 163-170, 2019.

[13] N. Mendes, M. Alves, R. Andrade, R. T. Ribeiro, A. L. Papoila, and F. Serrano, "Association between glycated haemoglobin, glycated albumin and fructosamine with neonatal birthweight and large-for-date status infants in gestational diabetes mellitus: a prospective cohort study," Journal of Obstetrics and Gynaecology, vol. 39, no. 6, pp. 768-773, 2019.

[14] M. Hod, A. Kapur, and H. D. McIntyre, "Evidence in support of the International Association of Diabetes in Pregnancy study groups'," American Journal of Obstetrics and Gynecology, vol. 221, no. 2, pp. 109-116, 2019.

[15] Z. Lv, L. Qiao, Q. Wang, and F. Piccialli, "Advanced machinelearning methods for brain-computer interfacing," IEEE/ ACM Transactions on Computational Biology and Bioinformatics, vol. 18, no. 5, pp. 1688-1698, 2021.

[16] A. Onmez, F. Gokosmanoglu, G. Baycelebi, and A. A. Arıkan, "Carotid Doppler ultrasonographic findings of dapagliflozin use in type 2 diabetic patients," The Aging Male, vol. 23, no. 5, pp. 1246-1250, 2020.

[17] K. W. Lee, S. M. Ching, V. Ramachandran et al., "Prevalence and risk factors of gestational diabetes mellitus in Asia: a systematic review and meta-analysis," BMC Pregnancy and Childbirth, vol. 18, no. 1, p. 494, 2018. 
[18] A. Familiari, C. Neri, C. Vassallo et al., "Fetal Doppler parameters at term in pregnancies affected by gestational diabetes: role in the prediction of perinatal outcomes," Ultraschall in der Medizin, vol. 41, no. 6, pp. 675-680, 2020.

[19] M. R. Babaei, M. Malek, F. T. Rostami, Z. Emami, N. H. Madani, and M. E. Khamseh, "Non-invasive vascular assessment in people with type 2 diabetes: diagnostic performance of Plethysmographic-and-Doppler derived ankle brachial index, toe brachial index, and pulse volume wave analysis for detection of peripheral arterial disease," Primary Care Diabetes, vol. 14, no. 3, pp. 282-289, 2020.

[20] Z. Wei, M. Mu, M. Li, J. Li, and Y. Cui, "Color Doppler ultrasound detection of hemodynamic changes in pregnant women with GDM and analysis of their influence on pregnancy outcomes," American Journal of Tourism Research, vol. 13, no. 4, pp. 3330-3336, 2021.

[21] G. de Fátima Ribeiro Dos Anjos, M. A. R. de Freitas, M. C. A. Dos Santos, and A. Diniz, "Doppler indices of the ophthalmic artery in pregnant women with pregestational diabetes mellitus with and without retinopathy," Journal of Ultrasound in Medicine, vol. 40, no. 2, pp. 251-257, 2021.

[22] M. Badarinza, O. Serban, L. Maghear et al., "Multimodal ultrasound investigation (grey scale, Doppler and 2D-SWE) of salivary and lacrimal glands in healthy people and patients with diabetes mellitus and/or obesity, with or without sialosis," Medical Ultrasonography, vol. 21, no. 3, pp. 257-264, 2019.

[23] L. Spatola and S. Andrulli, "Doppler ultrasound in kidney diseases: a key parameter in clinical long-term follow-up," Journal of Ultrasound, vol. 19, no. 4, pp. 243-250, 2016.

[24] M. Hoke, M. Schillinger, E. Minar, G. Goliasch, C. J. Binder, and F. J. Mayer, "Carotid ultrasound investigation as a prognostic tool for patients with diabetes mellitus," Cardiovascular Diabetology, vol. 18, no. 1, p. 90, 2019.

[25] A. Nickavar, B. Safaeian, H. Zaeri, H. Gharib, and T. Chaharnaei, "Usefulness of Doppler ultrasound for the early diagnosis of diabetic nephropathy in type 1 diabetes mellitus," Journal of Ultrasound, vol. 20, 2021. 